\title{
RIGIDITY THEOREMS FOR NONPOSITIVE EINSTEIN METRICS
}

\author{
ZHONGMIN SHEN
}

(Communicated by Jonathan M. Rosenberg)

\begin{abstract}
In this paper we study the following problem: When must a complete Einstein metric $g$ on an $n$-manifold with Ric $=(n-1) \lambda g, \lambda \leq 0$, be a metric of constant curvature $\lambda$ ?
\end{abstract}

\section{INTRODUCTION AND MAIN RESULTS}

Let $g$ be a complete riemannian metric on an $n$-manifold $M$. Denote by $R$ the curvature tensor of $g$. The Ricci curvature Ric is then defined as

$$
\operatorname{Ric}(x, y)=\sum_{i=1}^{n} g\left(R\left(x, e_{i}\right) e_{i}, y\right), \quad x, y \in T_{p} M,
$$

where $\left\{e_{1}, \ldots, e_{n}\right\}$ is an orthonormal basis for $T_{p} M$. The metric $g$ is said to be Einstein if the Ricci curvature is constant, i.e.,

$$
\operatorname{Ric}=(n-1) \lambda g
$$

for some constant $\lambda . \lambda$ is called the Einstein constant of $g$. It is clear that in dimension three the metric $g$ is Einstein with $\mathrm{Ric}=(n-1) \lambda g$ if and only if it has constant curvature $\lambda$, i.e.,

$$
R(x, y) z=\lambda(g(y, z) x-g(x, z) y), \quad x, y, z \in T_{p} M .
$$

In higher dimensions, this is not the case. One may ask if an Einstein metric has constant curvature whenever it has almost constant curvature in a certain sense. From now on we always assume that $g$ is a complete Einstein metric with Einstein constant $\lambda$. It is natural to consider the new tensor $\stackrel{\circ}{R}$, defined

Received by the editors January 8, 1991 and, in revised form, March 19, 1991.

1991 Mathematics Subject Classification. Primary 53C25; Secondary 53C20.

Key words and phrases. Einstein metrics, rigidity, Sobolev inequality, the first eigenvalue, diameter, volume.

Research at MSRI supported in part by NSF Grant DMS-8505550. 
by

$$
\stackrel{\circ}{R}(x, y) z=R(x, y) z-\lambda(g(y, z) x-g(x, z) y), \quad x, y, z \in T_{p} M .
$$

Denote by $\sigma$ the pointwise norm of $\stackrel{\circ}{R}$, defined by

$$
\sigma=\sqrt{\sum_{i j k l} g\left(\stackrel{\circ}{R}\left(e_{i}, e_{j}\right) e_{k}, e_{l}\right)^{2}},
$$

where $\left\{e_{1}, \ldots, e_{n}\right\}$ is an orthonormal basis for $T_{p} M$. By a formula in $[\mathrm{H}]$, one can easily show that $\sigma$ satisfies

$$
\Delta \sigma+c_{0}(n) \sigma^{2}-2(n-1) \lambda \sigma \geq 0
$$

in the sense of distribution, where $c_{0}(n)$ is a positive constant depending only on $n$ and $\Delta$ denotes the Laplace-Beltrami operator (in $\mathbb{R}^{n}, \Delta=\sum_{i=1}^{n} \partial^{2} / \partial x_{i}^{2}$ ).

In the case of $\lambda>0$, Myers's Theorem (cf., e.g., [CE]) tells us that the manifold is closed. Thus by integrating (1), one obtains

Theorem 1 (Berger [B]). Given $n$, there is a small constant $\varepsilon=\varepsilon(n)>0$ depending only on $n$ such that if a complete Einstein metric $g$, with $\lambda>0$, on an $n$-manifold satisfies $\sigma \leq \lambda \varepsilon$, then $\sigma \equiv 0$, i.e., $g$ has constant curvature $\lambda$.

In [S] the author gives an $L^{\frac{n}{2}}$-version of Theorem 1, which says that if

$$
\int \sigma^{\frac{n}{2}} \leq \lambda^{\frac{n}{2}} \operatorname{vol}(M) \varepsilon
$$

for some small $\varepsilon=\varepsilon(n)>0$, depending only on $n$, where $\operatorname{vol}(M)$ denotes the volume of $(M, g)$, then $\sigma \equiv 0$.

In the case of $\lambda \leq 0$, the manifold can be compact or noncompact. First let us consider the case $\lambda=0$. In this case, the following fact is known: There is a small constant $\varepsilon=\varepsilon(n)>0$ depending only on $n$, if a Ricci-flat metric $g$ on a closed $n$-manifold $M$ satisfies

$$
\sigma \cdot \operatorname{dia}(M)^{2} \leq \varepsilon
$$

where $\operatorname{dia}(M)$ denotes the diameter of $g$, then $\sigma \equiv 0$, i.e., $g$ is flat. The proof of this fact is trivial. By a theorem of Gromov [G], any almost flat manifold is aspherical, i.e., its universal cover is diffeomorphic to $R^{n}$. Thus for a sufficiently small $\varepsilon=\varepsilon(n)$, (2) implies that the universal cover $\widetilde{M}$ is diffeomorphic to $\mathbb{R}^{n}$. On the other hand, by the Cheeger-Gromoll's Splitting Theorem (cf. [CG]), $\widetilde{M}$ with the induced metric $\tilde{g}$ is isometric to a riemannian product $N \times \mathbb{R}^{k}$ for some closed riemannian manifold $N$. Thus $N$ must be a point and $\tilde{g}$ is flat. Therefore $g$ is flat. This argument in fact shows that all nonnegatively Ricci-curved aspherical manifolds are flat. By a theorem of Fukaya-Yamaguchi [FY], if $\operatorname{dia}(M) \leq D$, then condition (2) can be replaced by $-1 \leq K_{g} \leq \varepsilon$ for a small number $\varepsilon=\varepsilon(n, D)>0$, where $K_{g}$ denotes the sectional curvature of $g$.

In $\S 2$ we will prove an $L^{\frac{n}{2}}$-version of the above fact, that is, 
Theorem 2. Given $n$, there is a small constant $\varepsilon=\varepsilon(n)>0$ depending only on $n$ such that if a Ricci-flat metric $g$ on a closed $n$-manifold satisfies

$$
\int \sigma^{\frac{n}{2}} \leq \frac{\operatorname{vol}(M)}{\operatorname{dia}(M)^{n}} \varepsilon,
$$

then $\sigma \equiv 0$, i.e., $g$ is flat.

For complete Ricci-flat metrics on noncompact $n$-manifolds, some rigidity phenomena have been discovered (cf. [A2, Ba, S], etc.). Roughly speaking, if a complete Ricci-flat metric $g$ has sufficiently small total curvature, i.e., there is a small $\varepsilon=\varepsilon(n)>0$ such that if

$$
\int \sigma^{\frac{n}{2}} \leq \nu_{M}^{n+1} \varepsilon
$$

where

$$
\nu_{M}:=\lim _{r \rightarrow+\infty} \frac{\operatorname{vol}(B(p, r))}{\sigma_{n} r^{n}}>0,
$$

where $\sigma_{n}$ denotes the volume of the unit ball in $\mathbb{R}^{n}$, then $\sigma \equiv 0$. It is worth mentioning that the result of Anderson [A2] does not require (3), but requires that $\nu_{M} \geq 1-\varepsilon$ for a small $\varepsilon=\varepsilon(n)>0$.

Now let us consider the case of $\lambda<0$. The following theorem is first proved by $\mathrm{Ye}[\mathrm{Y}$, Theorem 2].

Theorem $3([\mathrm{Y}])$. Given $n, D>0$, and $\lambda<0$, there is a small constant $\varepsilon=$ $\varepsilon(n, \sqrt{-\lambda} D)>0$ such that if an Einstein metric $g$, with $\lambda<0$, on a closed $n$-manifold satisfies $\operatorname{dia}(M) \leq D$ and $\sigma \leq|\lambda| \varepsilon$, then $\sigma \equiv 0$, i.e., $g$ has constant curvature $\lambda$.

In $\S 3$ we will prove the following $L^{\frac{n}{2}}$-version of Theorem 3 .

Theorem 4. Given $n, D>0$, and $\lambda<0$, there is a small constant $\varepsilon=$ $\varepsilon(n, \sqrt{-\lambda} D)>0$ such that if an Einstein metric $g$, with $\lambda<0$, on a closed $n$-manifold satisfies $\operatorname{dia}(M) \leq D$ and

$$
\int \sigma^{\frac{n}{2}} \leq|\lambda|^{\frac{n}{2}} \operatorname{vol}(M) \varepsilon
$$

then $\sigma \equiv 0$, i.e., $g$ has constant curvature $\lambda$.

Complete Einstein metrics on noncompact $n$-manifolds with Einstein constant $\lambda<0$ are still not completely understood. The Sobolev inequalities do not hold on such manifolds. Instead, the Poincare inequalities hold, which will be used to prove the following

Theorem 5. Let $g$ be a complete Einstein metric on a noncompact simply connected $n$-manifold with $\lambda<0$. Suppose $n \geq 10$. There is a small constant $\varepsilon=\varepsilon(n)>0$ such that if

(i) $\sigma \leq|\lambda| \varepsilon$ and 
(ii) for some $p \in M$,

$$
\lim _{r \rightarrow+\infty} e^{-\delta_{n}|\lambda| \frac{1}{2} r} \int_{B(p, r)} \sigma^{2}=0,
$$

where $\delta_{n}=\frac{1}{3} \sqrt{(n-1)(n-9)}>0$ and $B(p, r)$ denotes the geodesic ball of radius $r$ around $p$, then $\sigma \equiv 0$, i.e., $g$ has constant curvature $\lambda<0$.

The proof of Theorem 5 will be given in $\S 4$. The author does not know the case of $n \leq 9$.

The author would like to thank S. Bando for bringing the problem of Theorem 5 to his attention. Thanks also to M. Anderson for many helpful discussions.

\section{Closed Einstein manifolds with $\lambda=0$}

In this section we will prove Theorem 2 . The argument given here is quite standard and similar to that given in Lemma 2.1 of [A1].

In $\S 1$ we have shown that every Ricci-flat metric satisfying (2) for some small $\varepsilon=\varepsilon(n)$ is flat. Throughout this section $M=(M, g)$ always denotes a closed Ricci-flat manifold of dimension $n \geq 4$ and $c_{i}(n)$ 's denote constants depending only on $n$. In the case of $\lambda=0,(1)$ is equivalent to

$$
\Delta \sigma+c_{0}(n) \sigma^{2} \geq 0
$$

in the sense of distribution. Recall that the following Sobolev inequality holds in $M$ (cf. [Be] for references):

$$
\|f\|_{\frac{2 n}{n-2}} \leq c_{1}(n) \operatorname{vol}(M)^{-\frac{1}{n}}\left[\operatorname{dia}(M)\|\nabla f\|_{2}+\|f\|_{2}\right]
$$

for every $f \in C^{\infty}(M)$.

Multiply (4) by $\sigma^{\alpha}$ for $\alpha \geq 1$. Integration by parts gives

$$
c_{0}(n) \int \sigma^{\alpha+2} \geq \frac{4 \alpha}{(\alpha+1)^{2}} \int\left|\nabla \sigma^{\frac{\alpha+1}{2}}\right|^{2} \geq \frac{1}{\alpha} \int\left|\nabla \sigma^{\frac{\alpha+1}{2}}\right|^{2} .
$$

Taking $f=\sigma^{\frac{\alpha+1}{2}}$ in (5), we obtain by (6)

$$
\left\|\sigma^{\frac{\alpha+1}{2}}\right\|_{\frac{2 n}{n-2}} \leq c_{2}(n) \operatorname{vol}(M)^{-\frac{1}{n}}\left[\alpha^{\frac{1}{2}} \operatorname{dia}(M)\left\|\sigma \cdot \sigma^{\alpha+1}\right\|_{1}^{\frac{1}{2}}+\left\|\sigma^{\frac{\alpha+1}{2}}\right\|_{2}\right] .
$$

Taking $\alpha+1=\frac{n}{2}$ in (7) and applying Hölder's inequality to $\left\|\sigma \cdot \sigma^{n / 2}\right\|_{1}$, we have

$$
\left\|\sigma^{\frac{n}{4}}\right\|_{\frac{2 n}{n-2}} \leq c_{3}(n) \operatorname{vol}(M)^{-\frac{1}{n}}\left[\operatorname{dia}(M)\|\sigma\|_{\frac{n}{2}}^{\frac{1}{2}}\left\|\sigma^{\frac{n}{4}}\right\|_{\frac{2 n}{n-2}}+\|\sigma\|_{\frac{n}{2}}^{\frac{n}{4}}\right] .
$$

It follows from (8) that there is a small constant $\varepsilon(n)>0$, such that if for some $\varepsilon \leq \varepsilon(n)$

$$
\|\sigma\|_{\frac{n}{2}} \leq \frac{\operatorname{vol}(M)^{\frac{2}{n}}}{\operatorname{dia}(M)^{2}} \varepsilon
$$

then

$$
\begin{aligned}
\|\sigma\|_{\frac{q}{2}} & =\left\|\sigma^{\frac{n}{4}}\right\|_{\frac{2 n}{n-2}}^{\frac{4}{n}} \leq c_{4}(n) \operatorname{vol}(M)^{-\frac{4}{n^{2}}}\|\sigma\|_{\frac{n}{2}} \\
& \leq c_{5}(n) \operatorname{vol}(M)^{\frac{2}{n}-\frac{4}{n^{2}}} \operatorname{dia}(M)^{-2},
\end{aligned}
$$


where $q=\frac{n}{2} \cdot \frac{2 n}{n-2}$. For general $\alpha \geq 1$, by Hölder's inequality, the interpolation inequality, and (10), we have that for all $\theta>0$

$$
\begin{aligned}
\left\|\sigma \cdot \sigma^{\alpha+1}\right\|_{1} & \leq\|\sigma\| \frac{q}{2}\left\|\sigma^{\frac{\alpha+1}{2}}\right\|_{\frac{2 q}{q-2}}^{2} \\
& \leq c_{5}(n) \operatorname{vol}(M)^{\frac{2}{n}-\frac{4}{n^{2}}} \operatorname{dia}(M)^{-2}\left(\theta\left\|\sigma^{\frac{\alpha+1}{2}}\right\|_{\frac{2 n}{n-2}}+\theta^{-\frac{n-2}{2}}\left\|\sigma^{\frac{\alpha+1}{2}}\right\|_{2}\right)^{2} .
\end{aligned}
$$

Thus it follows from (7) and (11) that

$$
\begin{aligned}
\left\|\sigma^{\frac{\alpha+1}{2}}\right\|_{\frac{2 n}{n-2}} \leq c_{6}(n)\left[\alpha^{\frac{1}{2}} \operatorname{vol}(M)^{-\frac{2}{n^{2}}} \theta\left\|\sigma^{\frac{\alpha+1}{2}}\right\|_{\frac{2 n}{n-2}}\right. \\
\left.+\left(\alpha^{\frac{1}{2}} \operatorname{vol}(M)^{-\frac{2}{n^{2}}} \theta^{-\frac{n-2}{2}}+\operatorname{vol}(M)^{-\frac{1}{n}}\right)\left\|\sigma^{\frac{\alpha+1}{2}}\right\|_{2}\right] .
\end{aligned}
$$

Choosing $\theta=\frac{1}{2} c_{6}(n)^{-1} \alpha^{-\frac{1}{2}} \operatorname{vol}(M)^{\frac{2}{n^{2}}}$, we obtain by (12)

$$
\left\|\sigma^{\frac{\alpha+1}{2}}\right\|_{\frac{2 n}{n-2}} \leq c_{7}(n) \alpha^{\frac{n}{4}} \operatorname{vol}(M)^{-\frac{1}{n}}\left\|\sigma^{\frac{\alpha+1}{2}}\right\|_{2} .
$$

Let $\chi=\frac{n}{n-2}$ and $\alpha+1=\frac{n}{2} \chi^{i}, i \geq 0$. It follows from (13) that

$$
\begin{aligned}
\|\sigma\|_{\frac{n}{2} \chi^{i+1}} & \leq c_{8}(n)^{\frac{1}{\chi^{i}}} \chi^{\frac{i}{x^{i}}} \operatorname{vol}(M)^{-\frac{4}{n^{2}} \cdot \frac{1}{\chi^{i}}}\|\sigma\|_{\frac{n}{2} \chi^{i}} \\
& \leq c_{8}(n)^{\frac{1}{\chi^{i}}+\cdots+\frac{1}{\chi^{0}}} \chi^{\left(\frac{i}{\chi^{i}}+\cdots+\frac{1}{\chi^{i}}\right)} \operatorname{vol}(M)^{-\frac{4}{n^{2}}\left(\frac{1}{x^{i}}+\cdots+\frac{1}{\chi^{0}}\right)}\|\sigma\|_{\frac{n}{2}} .
\end{aligned}
$$

Letting $i \rightarrow+\infty$, we obtain

$$
\sigma \leq c_{9}(n) \operatorname{vol}(M)^{-\frac{2}{n}}\|\sigma\|_{\frac{n}{2}} \leq c_{9}(n) \operatorname{dia}(M)^{-2} \varepsilon,
$$

i.e.,

$$
\sigma \cdot \operatorname{dia}(M)^{2} \leq c_{9}(n) \varepsilon
$$

The last inequality follows from (9). Choosing a smaller $\varepsilon$ in (9) if necessary, by the argument in $\S 1$, we conclude that $\sigma \equiv 0$, i.e., $g$ is flat. This completes the proof of Theorem 2 .

\section{Closed Einstein manifolds with $\lambda<0$}

In this section we will only give a sketch of the proof of Theorem 4 . The method applied here is very standard and similar to that given in $\S 2$. Let $M=$ $(M, g)$ be a closed Einstein $n$-manifold with Einstein constant $\lambda<0$ and $\operatorname{dia}(M) \leq D$. Throughout this section $c_{i}(n)$ 's always denote positive constants depending only on $n$.

First one has the following Sobolev inequality in $M$ (cf., e.g., [Be] for references): for every $f \in C^{\infty}(M)$

$$
\|f\|_{\frac{2 n}{n-2}} \leq c_{1}(n) C(\sqrt{|\lambda|} D)^{-1} \operatorname{vol}(M)^{-\frac{1}{n}}\left(|\lambda|^{-\frac{1}{2}}\|\nabla f\|_{2}+\|f\|_{2}\right),
$$

where $C(x), x>0$, is the unique positive root of the equation

$$
y \int_{0}^{x}(\cosh t+y \sinh t)^{n-1} d t=\int_{0}^{\pi} \sin ^{n-1} t d t .
$$


Similarly, by (1) and (14) we obtain that there is a constant $\varepsilon(n)>0$ if for some $\varepsilon \leq \varepsilon(n) C(\sqrt{|\lambda|} D)^{2}$

$$
\|\sigma\|_{\frac{n}{2}} \leq|\lambda| \operatorname{vol}(M)^{\frac{2}{n}} \varepsilon
$$

then for $q=\frac{n}{2} \cdot \frac{2 n}{n-2}$

$$
\begin{aligned}
\|\sigma\| \frac{q}{2} & \leq c_{2}(n) C(\sqrt{|\lambda|} D)^{-\frac{4}{n}} \operatorname{vol}(M)^{-\frac{4}{n^{2}}}\|\sigma\|_{\frac{n}{2}} \\
& \leq c_{3}(n) C(\sqrt{|\lambda|} D)^{2-\frac{4}{n}}|\lambda| \operatorname{vol}(M)^{\frac{2}{n}-\frac{4}{n^{2}}}
\end{aligned}
$$

and for $\alpha \geq 1$,

$$
\left\|\sigma^{\frac{\alpha+1}{2}}\right\|_{\frac{2 n}{n-2}} \leq c_{4}(n) C(\sqrt{|\lambda|} D)^{-1} \alpha^{\frac{n}{4}} \operatorname{vol}(M)^{-\frac{1}{n}}\left\|\sigma^{\frac{\alpha+1}{2}}\right\|_{2} .
$$

Then the last argument in $\S 2$ carries over to give

$$
\sigma \leq c_{5}(n) C(\sqrt{-\lambda} D)^{-2}|\lambda| \varepsilon .
$$

Choosing a smaller $\varepsilon$ in (15) if necessary, by Theorem 3 (Ye), one concludes that $\sigma \equiv 0$, i.e., $g$ has constant curvature $\lambda<0$.

\section{Proof of Theorem 5}

Let $M=(M, g)$ be a complete $n$-manifold. Denote by $\lambda_{1}(M, g)$ the first eigenvalue of $M$, defined as

$$
\lambda_{1}(M, g)=\inf \frac{\int|\nabla f|^{2}}{\int f^{2}},
$$

where the infumum is taken over all $f \in C_{0}^{\infty}(M)$ with compact support in $M$. It is proved in [M] that if $M$ is simply connected with sectional curvature $K_{g} \leq-\Lambda^{2}(\Lambda>0)$,

$$
\lambda_{1}(M, g) \geq \frac{(n-1)^{2}}{4} \Lambda^{2},
$$

i.e., for every $f \in C_{0}^{\infty}(M)$,

$$
\frac{1}{4}(n-1)^{2} \Lambda^{2} \int f^{2} \leq \int|\nabla f|^{2} .
$$

From now on $(M, g)$ always denotes a complete Einstein $n$-manifold with Einstein constant $\lambda<0$ and $c_{i}(n)$ 's denote positive constants depending only on $n$. Clearly, there is a small constant $\varepsilon(n)>0$ such that if for some $\varepsilon \leq \varepsilon(n)$, $\sigma \leq|\lambda| \varepsilon$, then the sectional curvature satisfies

$$
K_{g} \leq-\left(1-c_{1}(n) \varepsilon\right)|\lambda|<0 .
$$

By (1) and (17) we have

$$
\Delta \sigma+\left(c_{0}(n) \varepsilon+2(n-1)\right)|\lambda| \sigma \geq 0 .
$$

Multiply (18) by $\sigma \eta^{2}$, where $\eta$ is a cut off function of compact support in $M$. Integration by parts gives

$$
\left(c_{0}(n) \varepsilon+2(n-1)\right)|\lambda| \int(\sigma \eta)^{2} \geq \int|\nabla(\sigma \eta)|^{2}-\int|\nabla \eta|^{2} \sigma^{2} .
$$


Taking $f=\eta \sigma$ in (16), by (17) we have

$$
\frac{1}{4}(n-1)^{2}\left(1-c_{1}(n) \varepsilon\right)|\lambda| \int(\sigma \eta)^{2} \leq \int|\nabla(\sigma \eta)|^{2}
$$

so that by (19)

$$
\int|\nabla \eta|^{2} \sigma^{2} \geq\left[\frac{1}{4}(n-1)(n-9)-c_{2}(n) \varepsilon\right]|\lambda| \int(\sigma \eta)^{2} .
$$

Now suppose $n \geq 10$. Take $\delta(n)=\frac{1}{3} \sqrt{(n-1)(n-9)}$. One can choose a smaller $\varepsilon$ in (17) if necessary, such that

$$
\frac{1}{4}(n-1)(n-9)-c_{2}(n) \varepsilon \geq \frac{1}{4} e^{2} \delta(n)^{2} .
$$

Choosing $\eta(x)=\eta(d(p, x))$, where

$$
\eta(t)= \begin{cases}1 & \text { if } t \leq r, \\ \frac{R-t}{R-r} & \text { if } r \leq t \leq R, \\ 0 & \text { if } t \geq R,\end{cases}
$$

we obtain by $(20)$

$$
\frac{1}{(R-r)^{2}} \int_{B(p, R)} \sigma^{2} \geq \frac{1}{4} e^{2} \delta(n)^{2}|\lambda| \int_{B(p, r)} \sigma^{2}
$$

For any $r_{0}>0$, take $r_{j}=2 \delta(n)^{-1}|\lambda|^{-\frac{1}{2}} j+r_{0}, j \geq 0$. It then follows from (21) that

$$
\int_{B\left(p, r_{j}\right)} \sigma^{2} \geq e^{2} \int_{B\left(p, r_{j-1}\right)} \sigma^{2} \geq e^{2 j} \int_{B\left(p, r_{0}\right)} \sigma^{2}=e^{\delta(n)|\lambda|^{\frac{1}{2}}\left(r_{j}-r_{0}\right)} \int_{B\left(p, r_{0}\right)} \sigma^{2} .
$$

Thus it is easy to see that

$$
\int_{B\left(p, r_{0}\right)} \sigma^{2} \leq e^{-\delta(n)|\lambda|^{\frac{1}{2}}\left(r_{j}-r_{0}\right)} \int_{B\left(p, r_{j}\right)} \sigma^{2} .
$$

Letting $r_{j} \rightarrow+\infty$, by Theorem $5(\mathrm{ii})$, one obtains $\sigma \equiv 0$ on $B\left(p, r_{0}\right)$. Since $r_{0}$ is arbitrary, one concludes that $\sigma \equiv 0$ on $M$, i.e., $g$ has constant curvature $\lambda<0$. This completes the proof of Theorem 5 .

\section{REFERENCES}

[A1] M. Anderson, Ricci curvature bounds and Einstein metrics on compact manifolds, J. Amer. Math. Soc. 2 (1989), 455-490.

[A2] - Convergence and rigidity of manifolds under Ricci curvature bounds, Invent. Math. 102 (1990), 429-446.

[B] M. Berger, Sur les variétés d'Einstein compactes, C. R. de la III ${ }^{0}$ réunion du groupement des mathématiciens d'expression Latine, Namur, 1965, pp. 35-55.

[Ba] S. Bando, Bubbling out of Einstein manifolds, Tohoku Math. J. 42 (1990), 205-216.

[Be] P. Berard, From vanishing theorems to estimating theorems: the Bochner technique revisited, Bull. Amer. Math. Soc. 19(1988), 371-406. 
[CE] J. Cheeger and D. Ebin, Comparison theorem in Riemannian geometry, North-Holland, Amsterdam, 1975.

[CG] J. Cheeger and D. Gromoll, The splitting theorem for manifolds of nonnegative Ricci curvature, J. Differential Geom. 6 (1971), 119-128.

[FY] K. Fukaya and T. Yamaguchi, Almost nonpositively curved manifolds, J. Differential Geom. 33 (1991), 67-90.

[G] M. Gromov, Almost flat manifolds, J. Differential Geom. 13 (1978), 231-241.

[H] R. Hamilton, Three-manifolds with positive Ricci curvature, J. Differential Geom. 17 (1982), 255-306.

[M] H. P. Mckean, An upper bound to the spectrum on a manifolds of negative curvature, $\mathbf{J}$. Differential Geom. 4 (1970), 359-366.

[S] Z. Shen, Some rigidity phenomena for Einstein metrics, Proc. Amer. Math. Soc. 108 (1990), 981-987.

[Y] R. Ye, Ricci flow, Einstein metrics and space forms, preprint, 1990.

Mathematical Sciences Research Institute, Berkeley, California 94720

Current address: Department of Mathematics, University of Michigan, Ann Arbor, Michigan 48109-1003

e-mail: zhongmin@math.lsa.umich.edu 